\title{
A UNIQUE DATA VALIDATION PROCESS BY INTERNAL AND EXTERNAL QUALITY ASSURANCE SYSTEM AIMED TO COMBAT RESEARCH MALPRACTICE.
}

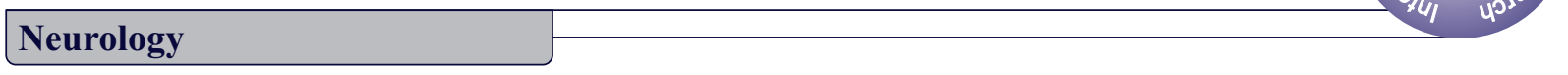

Mr. Sushant Kaushal

Dr. Rahul Tyagi
M.Tech Neuroscience Research Lab, Department of Neurology, Postgraduate Institute of Medical Education and Research, Chandigarh

Ph.D Neuroscience Research Lab, Department of Neurology, Postgraduate Institute of Medical Education and Research, Chandigarh

Post-Doctoral Fellow Department of Pharmacology, Emory University School Of Medicine, Atlanta

MSC Neuroscience Research Lab, Department of Neurology, Postgraduate Institute of Medical Education and Research, Chandigarh

Ms. Priya Battu

MSC Neuroscience Research Lab, Department of Neurology, Postgraduate Institute of Medical Education and Research, Chandigarh

Ms. Priya Mehra

Dr. Neel Kamal Sharma

Mr. Kaushal Sharma

Post-Doctoral Fellow Armed Forces Radiobiology Research Institute, Bethesda.

Ph.D Neuroscience Research Lab, Department of Neurology, Postgraduate Institute of Medical Education and Research, Chandigarh

Prof. Akshay Anand*

Ph.D Neuroscience Research Lab, Department of Neurology, Postgraduate Institute of Medical Education and Research, Chandigarh *Corresponding Author

\section{ABSTRACT}

Research fabrication and data fraud are one of the major concerns worldwide which are rising and evident from increasing number of retractions in peer reviewed Journals. If not checked, this can impact the reputation of a research organisation as well as the costs of translation of research data. In basic research extremely publicized cases of falsification of data have been reported and it is possible, that there are many unreported or undetected cases. In order to improve the quality standards, the validation processes were implemented for data quality at Neuroscience research lab in India for verifying $\mathrm{PhD}$ thesis results. Good Laboratory practices could be implicated in every research institute so that data impostures were prevented. We aimed to establish superior data quality by randomly verifying raw data in multiple projects funded by national agencies before publication of results at Neuroscience Research Lab.

\section{KEYWORDS}

Quality Research, GLP, Validation, Neuroscience, Intra-laboratory validation, Standard Operating Procedures, checklist.

\section{INTRODUCTION}

Since 1976, Good Laboratory Practices were implemented which determined to establish accurate documentation, quality check and undeviating data. GLP was introduced to monitor the compliance of non-clinical safety testing of drugs at first in the USA in order to regulate the generation of fraudulent data and unethical practices(Carson \& Dent, 2007). GLP principles were formulated by Organisation of Economic Co-operation and Development [OECD] for global compliance of testing facilities(Kiranmai) but not research facilities.

The evidence based practice were soon acquiring a tremendous amount of significance in research based laboratories where cross sectional studies generate research data which remains non-validated even after publication, due to lack of routine checkups, recall of record maintenance etc. (Wassie, Zeleke, Dachew, \& Kebede, 2017). Scientists believe that Good Clinical Laboratory Practice [CGLP] basically ensures compliance to the control of disastrous effects in the field of research and how to implement good science. On the contrary, these systems provide for an evidence based validation of studies to ensure the audit of data thus enhancing the transparency of work. However, in the last 60 years, Research and Development have gained many breakthroughs in instrumentation which may have a positive impact on quality and quantity of research (Jena \& Chavan, 2017) as validation improves the scientific quality of data. The case of testing laboratories adopting the implementation of GLP results in the effectiveness of results thus making the patients more confident about the quality of the diagnostics and treatment (Horvath, 2013)

As mentioned above, the basic research investigations were never monitored by the GLP systems or any other alternative quality assurance module (Carson \& Dent, 2007). Quality assurance is a challenging task for implementation in research ecosystem for developing countries because it requires consistency, manpower and resources without Institutional incentive for its implementation because the data duplication, plagiarism and cases of data theft are major factors which brought the research from developing countries under the radar. These factors partly prevented the research from developing countries to be published in reputed journals.

The Neuroscience Research Lab at PGIMER, Chandigarh, India [hereafter denoted as NRL] have voluntarily implemented GLP in basic research lab, hitherto never implemented in world, to meet the globally accepted quality standards and in order to bridge the chasm that exists between the quality systems in labs from developing and developed countries. The initial implementation included creation of validation documents, procedures and an independent review mechanism through a quality assurance unit in order to meet the quality requirements of research studies. As a result the facility was recognized by Quality Council of India [QCI] under Research category for this case study "Redefining Quality standard in basic research investigations by broadening the purview of Good Laboratory Practices" (Horvath, 2013) and later "Digital research lab for enhancing capability: Towards skill development and community outreach" (Neuroscience Research Lab \& Medical Education and Research, 2016). The recognition of these systems has made it convenient to expand the systems and implement the tools for backtracing the data resulting in a $\mathrm{PhD}$ thesis, as a case study.

\subsection{Requirement of Thesis validation:}

Truthfulness and morality are basic principles of research. Adoption of these principles is important for the development of science and 
community awareness. Any deviations from these ethics are considered fraud or scientific misconduct. We therefore, intended to extend the available GLP module for validation of compliance to data generation scheme of GLP including compilation processes, digitizated storage, data analysis, cost audit and publication through various procedures and modules (fig1). This is consistent with other corporate houses and certain testing labs(Paszko \& Turner, 2001)

Equipment like autoclaves, pipettes, water bath, gel doc, microscopes, and refrigerators, PCRs were often validated for adherence to annual calibration and daily use entries as per established norms (Panel, 2012). Whether the process of experimental set up correlates with accurately labelled and periodically adopted Quality Assurance procedures by QA in-charge, was determined along with periodic audits in testing labs(Sickles, 1992). Similarly, the corresponding use of chemical and reagents sample log was verified with proper labelling of reagent bottle used for the purpose, like good labs(Cushman, Cornell, Howard, Bovill, \& Tracy, 1995). The documentation of test facility was checked for accuracy. These were evidenced properly and quality assurance whose protocol has been approved by the Study Director.

\section{METHODS}

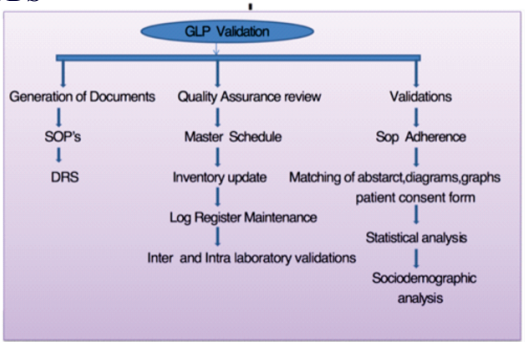

Fig 1.Flowchart showing the methods of research data validation

\subsection{Validation of documents for Data Acquisition}

The documents pertaining to data acquisition, verification and validation were prepared by the $\mathrm{PhD}$ students according to the Plan of Thesis, in consultation with the Study Director. The documents were got verified and finally submitted to the Quality Assurance cell(Hancock \& Algozzine, 2016). These documents included Standard operating Procedures[SOPs] for each experiment, Data Recording sheets [DRSs] for all experiments, Raw Book, checklists, Monthly Master Schedules [MS] throughout the duration of PhD, Log registers of chemicals used in experiments, indexed $\mathrm{PhD}$ result folders, Sample logs for sample used, documents for inventory management, server storage records of digital data etc(Refaeilzadeh, Tang, \& Liu, 2016)

\subsubsection{DRS [Data Record Sheet]}

Data recording sheets were filled for the real time documentation of correctness/deviations as per GLP rules (Haider, 2001). DRS were issued through Quality Assurance [QA] cell for stipulated time duration. In the event of change in the experimental procedures, DRSs were modified with compliance to the QA approval. DRSs were coded as per master coding used in the established GLP module [fig 2]. These DRSs provided an important tool to verify and validate the experimental dates with chemical log sheets and the corresponding experiments. A typical DRS would contain the step by step information regarding the experimental protocol performed regularly/periodically at NRL [fig2]. The DRS also recorded the materials and equipment used in the experiment in order to maintain back traceability of the usage in their respective usage log books as per estab

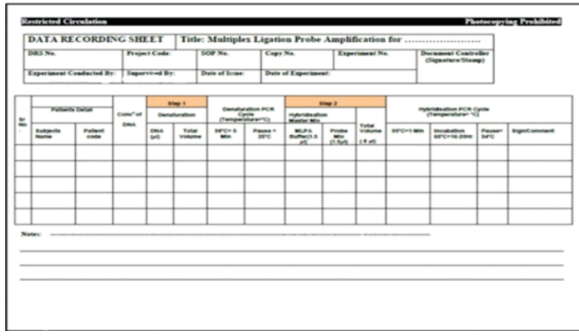

lished norms (Opara, 2003)

Fig.2 Data recording sheet

\subsubsection{SOP's [Standard Operating Procedures]}

Standard Operating Procedures were documented protocols and the backbone of Good Laboratory Practices. These were periodically used to work for maximum safety and operational efficiency and to reduce the chance of errors(Hartmann-Fritsch, Marino, \& Reichmann, 2016) To validate the experiment protocols, the SOPs pertaining to the $\mathrm{PhD}$ Thesis plan [fig 3], were formulated by the researchers followed by validation by Study Director. SOPs, for thesis 1 including experimental [Morris water maze, cDNA and RNA synthesis, DNA isolation, ERG, Stem cell isolation, Laser Doppler, Immunohistochemistry, MLPA Plasma isolation, Tunnel assay etc.], academic [Journal club, DC, DDC, Seminar], SOP's for thesis 2 [ SNP genotyping, ELISA, Total protein estimation [By Bradford method] linear range, Flow cytometry of mononuclear lymphocytes, DNA isolation, Separation of lymphocytes and serum] and administrative [Ethical clearance, ordering material, Joint grant requirements, accounts] procedures were formulated. Each SOP was master coded as per GLP module by the personnel of the Quality assurance cell. Format of SOP was obtained from SOP of SOPs.

Restricted Circulation Photocopving Prohibite

\section{STANDARD OPERATING PROCEDURI}

\begin{tabular}{|c|c|c|c|c|}
\hline SOP No. & $\begin{array}{c}\text { Edition No. } \\
01\end{array}$ & Effective Date & Review Date & $\begin{array}{l}\text { Document Controller } \\
\text { (Signature/Stamp) }\end{array}$ \\
\hline Copy No. & Date of Issue & Location ( & nit/Division) & \\
\hline
\end{tabular}

Fig.3 An example of Standard operating procedure generated for a method regularly practiced in the laboratory

\subsubsection{Data archiving}

In the lab continuous monitoring systems were used for the storage of data with proper coding. The archive storage conditions had been predefined to maintain the integrity and sustainability of data, all environmental monitoring procedures are implemented within archive storage with defined standard operating procedures in the designated area of storage. Access to the archive is only controlled by and restricted to the QA and the Study Director(Crouse, Coverston, \& Cychosz, 1998) A researcher had generated a request to the QA to obtain the archived data with a QA $\log$ mentioning date and sign at the time of issue and return.

\subsubsection{VPN [Virtual Private Network]}

Internet access is primarily associated with GLP to access data remotely for analysis and maintaining confidentiality of such data is achieved by VPN which is used in Neuroscience Research Lab for secured networking for remote access, storage and transferring online data(Corti, Van den Eynden, Bishop, \& Woollard, 2014; Furht \& Escalante, 2010). Each staff and student in Neuroscience Research Lab is assigned a server domain to store the confidential data with the remote access by Principal Investigator. VPN storage locations are also mentioned in the DRS to link the result data to its respective experiment and also for future access. The Firewall internet security is maintained which creates interface between other network and lab network.

\section{QUALITY ASSURANCE REVIEW}

Quality assurance cell was established independently to verify the quality and deviations of procedures providing the support for carrying out the validation processes as cited in various articles(Taylor \& Tranter, 1987) In NRL, QA analysis was carried out for every individual by the Quality In Charge at the end of every month which includes experimental details, adherence to self -declared Master Schedules, Purchase benchmarks, Inventories checking etc, as an adaptation to new amendments. The QA report also records the accomplishments and deviation from the Master Schedule and an explanation from the researcher to justify the deviation and include any unfinished work to be assigned into the next master schedule.

\subsection{Master Schedules}

Master schedules was working timeline of every individual research scholar typically for a month which was needed to be self-declared before starting of the month to QA under intimation to the Study Director(Evans \& Lindsay, 2013). The master schedule helps the researchers to plan their work schedule, book any high work load equipment, if needed and maintain their inventory required for the procedures, in advance and useful for effective planning and efficient 
execution QA evaluated the progress of every staff at the end of every month and made a report of the ongoing things and work which is not completed yet.

\subsection{Inventory update}

All inventories of lab were divided among the students who look after the usage of every material. Lab materials like reagents, pipettes, and glassware are placed on different shelves and refrigerators. A Concerned student in-charge of the inventory is responsible for proper usage of materials and periodic procurement through wish lists whenever items reach their critical level. Master inventory sheets are placed in the front of every shelf, cabinet and fridge for easy access. It was mandatory to update these sheets quarterly by the responsible student and should thereafter be reviewed by Quality Assurance in charge. These were also used by the validation processes.

\subsection{Checklists}

Checklists were prepared with lists of do's and don'ts for every task, ranging from seminar presentation to thesis writing, in order to ensure the completeness of the task without unreported deviation. Other examples include checklist for synopsis preparation, synopsis approval, seminar and Journal club presentation, Ethical clearance, manuscript writing etc. It helps to create a system dependent approach(Hooijmans, de Vries, Leenaars, Curfs, \& Ritskes-Hoitinga, 2011) which is another media for assessment of adherence profile of a student undergoing validation.

\subsection{Log register maintenance}

Sustenance use of log registers was mandatory task for every research scholars in the lab to maintain his/her record related to research activities. A log register maintains the record of Journal clubs presented and attended, leave records, literature search records, SOP records, workshops attended, record of personal discussion with PI and lab meeting attended etc. These records help to maintain the progress and evaluation of individual scholar at NRL.

\section{MIDTERM REVIEW}

In midterm verification Inter and Intra laboratory validations were done quarterly to ensure the efficacy of work. It was done by internal Quality Assurance in charge and outside laboratories like CDFD Hyderabad, India [fig 4].

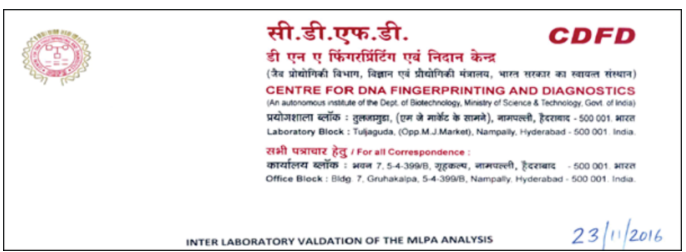

Fig 4. Copy of an Intra laboratory validation report for one of the procedures regularly used in the laboratory

\subsection{Physical verification}

Biannual audit of test facility by physical verification of inventories, sample $\log$, a log book was also carried out by external auditors to verify the GLP compliance of the students. Thus, it was mandatory for every scholar to update all the required data from time to time. Whether these procedures were followed or not it was also not analysed by the person who carried out the validation.

\section{Validation of acquired and analysed data before Thesis submission}

Validation of thesis was done in a curative way to analyse the thesis for its authenticity. A team of students were made by Principle Investigator to cross verify the data and some of the procedures explained above(Herr \& Anderson, 2014). The data was analysed on the basis of the following criteria: raw data and excel sheet, patient data to informed consents, Patient details to physical location and amount of sample left and so on.

5.1. Checking of Thesis Format -For writing a thesis, a proper protocol with guidelines was followed in a particular sequence which are- Abstract, Introduction, Review of literature Hypothesis, aim, objectives, result, conclusion etc. All these sections were reviewed for adherence to the format.

\subsection{Diagram and figures validation- Various graphs and}

illustrations, if any, were matched with relevant data which was cross checked to ensure that no irrelevant data was found filed. Citations were cross verified. The figures were closely matched with their respectively recorded DRS copies, raw book entries and VPN data archives to validate their authenticity and accuracy.

5.3. Socio demographic analyses - In socio demographic analyses, the telephonic verification was done by calling every individual patient and controls to match his/her data with excel sheet. For example, cross validation of the patient sleeping time, water intake capacity, diet, parent's education level and income etc. were cross checked.

5.4 Physical verification of samples-Each sample was verified for its $\log$, proper location, storage and coding to ensure if it was maintained properly and matched patient's details and date of sampling. It was also ensured that it was properly entered in its respective inventory.

5.5 Sample coding- Sample coding was carried out to code the sample for blinding and tracing the location and to maintain the sample log. The sample coding of various samples was verified as per established norms (Grant \& MacDonald, 1986)

5.6 Genetic result matching-The reports of genetic data and mutations of specific genes of patients on excel sheets were cross checked with hard copies. Results obtained from the capillary electrophoresis were also cross verified. Result verification of genetic data of patient with Dystrophin gene and other genes like APP, PSEN was enumerated and matched the hard copies with excel sheets in $\mathrm{PhD}$ thesis 1 and in another $\mathrm{PhD}$ thesis the same data of patients with Age related macular degeneration were analyzed and matched with hardcopy.

5.7 Consent form- Informed consent forms along with audio visual consents, Patient information sheets, Patient records were cross verified for validation along with signatures of witnesses(PaascheOrlow, Taylor, \& Brancati, 2003)

5.8 DRS [Data Record Sheet] -All DRS of experiments was cross examined to check if they were filled concurrently with the experiment. The process was done for locating any discrepancy or deviations in the experiment.

5.9 Neuropsychology of Patients - Neuropsychological assessment data was cross checked in the patient, control and follow-ups of a particular time period before archiving the data after coding. The data was tallied with attached consent form along with videography record.

5.10 Genetic result verification -Result verification of genetic data of patient with Dystrophin gene and other genes like APP, PSEN was enumerated and matched the hard copies with excel sheet.

5.11. Blinding sheet - Blinding sheet was prepared during data acquisition and analysis with every set of experiment. We checked if blinding sheets were attached with every data and the same was recorded.

\section{RESULTS}

A final report was prepared after $\mathrm{PhD}$ Thesis validation. The concerned Quality Assurance personnel wrote a GLP compliance statement indicating assurance for the validated data. A final master validation sheet was prepared with proper recommendations for errors.

\subsection{Documentation analysis}

The adherence to SOPs, matching of raw data to excel sheets, accuracy of raw data and statistical analysis of the data was done independently by using validation SOP. It was found that indexes were not made. Some discrepancies were found which were recorded and updated as per GLP compliance.

\subsection{Telephonic verification of patients}

$\mathrm{In} \mathrm{PhD}$ thesis 1- The data acquisition dates in records were matched with those obtained telephonically by contacting individuals recruited in the study data. The data of 90 male patients with an average age of 10-11 years were telephonically verified. In continuation to the validation process, the socio-demographic data verification was carried out by matching of hard copies with excel sheets corresponding to the telephonically obtained information including water intake, physical status, educational qualification, parents income, ambulation 
age, use of electronic devices, time of Neuropsychology and their follow ups etc. It was reported that 5 of the DMD cases had expired while telephone numbers of 9 patients did not match with records after 4 years of recruitment. Other minor errors were also found in the data which were included in the master validation sheet for further compliance and necessary correction [Fig5]

In $\mathrm{PhD}$ thesis 2- $280 \mathrm{AMD}$ patients and 200 controls were recruited in the study. The study consisted of participants [both AMD patients and controls] falling in the age group of 50 and above. During collection of Socio-demographic data, information pertaining to smoking habits, drinking habits, night sleeping hours, physical activity, yoga practice, history of surgery, co-morbidities etc was gathered. To cross validate the collected data, participants were contacted at their telephone numbers given by them during data collection. Patients were again asked similar questions on phone call. Cross-checking was done to match this data with already existing information. In addition, validation exercise also helped in checking whether participants have voluntarily participated in the study or not. A sheet was made enlisting number of patients who attended the call, 12 of them who didn't respond the call and 6 patients had died and 9 patients had invalid contact number [Table1]

\subsection{Neuropsychology Record}

In $\mathrm{PhD}$ thesis 1 - The Neuropsychology data was validated by preparing name wise patient list. Consent forms of patients attached with forms was checked individually. Consent forms were found to be attached with every questionnaire duly signed by witness.

\subsection{Matching of Experimental data}

In $\mathrm{PhD}$ thesis 2 - The experimental data comprised of ELISA and SNP genotyping data. The results were maintained in form of excel sheet. The samples were matched with raw data; Data recording Sheet [DRS] was checked to verify the validity of experiments [Fig 6]

Table 1 Thesis 2 Validation of various parameters and action take

\begin{tabular}{|l|l|l|l|l|}
\hline Parameter & $\begin{array}{l}\text { According to } \\
\text { concerned person }\end{array}$ & Observation & Correction made & Outcome \\
\hline $\begin{array}{l}\text { Calling } \\
\text { patients }\end{array}$ & $\begin{array}{l}\text { All the participants } \\
\text { had voluntarily } \\
\text { participated and socio- } \\
\text { demographic data was } \\
\text { correctly entered }\end{array}$ & $\begin{array}{l}\text { Some patients didn't pick } \\
\text { up the phone some wer } \\
\text { dead but for those we } \\
\text { were able to talk Socio- } \\
\text { demographic data entered } \\
\text { was correct }\end{array}$ & $\begin{array}{l}\text { Consent forms were checked for voluntary } \\
\text { participation of patients and it was made } \\
\text { sure that those forms had signature of } \\
\text { participants }\end{array}$ & $\begin{array}{l}\text { Finally those } \\
\text { participants were } \\
\text { recruited who had } \\
\text { matched socio- } \\
\text { demographic data or } \\
\text { signed consent forms }\end{array}$ \\
\hline $\begin{array}{l}\text { Physical verification of } \\
\text { samples }\end{array}$ & $\begin{array}{l}\text { All samples recruited } \\
\text { in study are physically } \\
\text { present }\end{array}$ & $\begin{array}{l}\text { Sample 259 not found } \\
\text { was maintained }\end{array}$ & $\begin{array}{l}\text { Wrong data entered by mistake was } \\
\text { removed } \\
\text { some patients was } \\
\text { missing }\end{array}$ & $\begin{array}{l}\text { Samples except 259 } \\
\text { was included }\end{array}$ \\
\hline Clinical data matching & $\begin{array}{l}\text { Clinical data record to procure clinical records } \\
\text { Consent forms were } \\
\text { present but some didn't } \\
\text { have signature of PI or } \\
\text { Ophthalmologist }\end{array}$ & $\begin{array}{l}\text { Incompleted } \\
\text { after getting clinical } \\
\text { records }\end{array}$ & Incompleted \\
\hline $\begin{array}{l}\text { Consent form matching } \\
\text { Experimental data }\end{array}$ & $\begin{array}{l}\text { All consent forms } \\
\text { were present } \\
\text { matches the excel } \\
\text { entries }\end{array}$ & Matched & Matched & $\begin{array}{l}\text { Matched entries } \\
\text { included }\end{array}$ \\
\hline
\end{tabular}

\section{DISCUSSION}

Cross validation of $\mathrm{PhD}$ Thesis by recording various parameters like telephonic verification, record of chemical usage with dates of experiments, thesis format, result cross verification, genetic data validation ensured validity and accuracy of the Thesis which was reassuring for both student and the Supervisor. The thesis of both $\mathrm{PhD}$ scholars were submitted after all compliances and corrections and Quality practises had been conducted. This instil a sense of responsibility and enhances accountability of data (McLachlan, 2017). Error reporting at the time of thesis validation, after following Quality practices, enhances the translation value of the research without further need to repeat experiments. Good Laboratory Practices and such Quality checks necessitate the research scholars to maintain the records including sample logs, master sample location chart etc. Format checking of Thesis showed the occurrence of proper algorithm which also reduces the time taken by supervisor to ensure minimising the deviations in the GLP compliance. DRSs, however, are a good source of real time deviation identifier along with proof of experimentation and corresponding utilization of chemicals. These are often installed in other establishments to ensure transparency and
6.5 Result Validation and sample location

Genetic result data was verified by following different steps. All the genetic data [coafflyser report, soft genetics reports] was attached with Neuropsychology data as analysed in Fig 5.

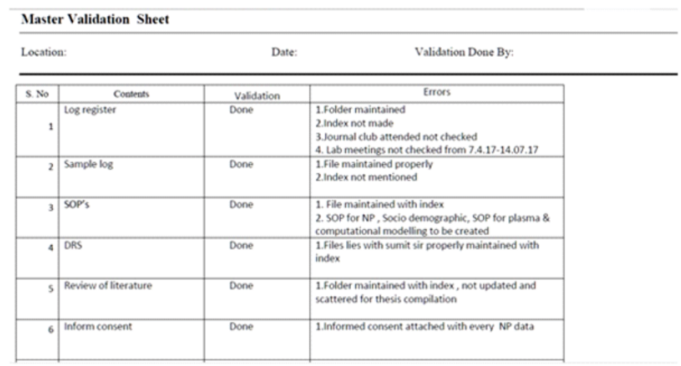

Fig 5. Thesis- 1 A copy of Master validating sheet reporting errors identified in a scholar's thesis data.

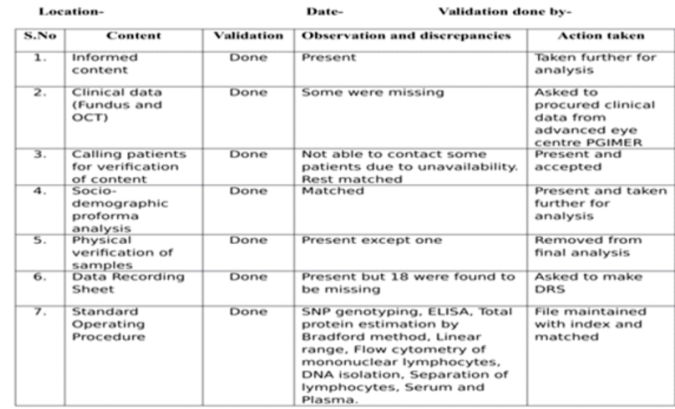

Fig6. Thesis 2- Master validating sheet reporting errors and action taken identified in a scholar's thesis data

increase cost effectiveness(Best \& Kahn, 2016). Similarly, maste schedules are used to steer the performance of researcher in a planned manner ensuring real time monitoring. Using VPN networks, the working of staff to becomes system dependent thus enhancing efficiency(Kerzner \& Kerzner, 2017). Socio-demographic data validation provided a proper record of patients with change in their habits over the time which necessitates follow ups for prospective studies. The critical aspect of Good Laboratory Practices requires proper audits and verifications which is used to empower data accuracy leading to valuable research. It is difficult to state whether this degree of compliance could be found in other labs not following GLP for the sheer reason that no lab in India and perhaps world follows GLP in the research settings(Organization, 2010)

These findings provide support to the current ideas suggesting the benefits of Good Laboratory Practices for the $\mathrm{PhD}$ thesis validation. Documentation in research is aimed to maintain the record of research for Quality Assurance thereby addressing of questions that may be asked under the Right to Information, a right given by all Indians constitution. The validated data enables accurate reporting of data 
manuscripts to various Journals, attracting high citations, awards, grants and national projects especially when no lab in the country follows similar benchmarks implemented.

\section{CONCLUSION AND FUTURE PROSPECTS}

Today research misconduct and data falsification is a serious threat to the credibility of science. The manipulated research publications can disrupt the basic scientific honesty and can mislead a research group. It may also wrongly extrapolate the data for community. A 2009 report published by the Office of Research Integrity [ORI] of the US Department of Health and Human Services mentioned a large number of research misconduct analysed from opened cases from 2007-08 publications. A majority of the cases were found to be involved with image manipulation(Mayer \& Steneck, 2007). A gradual increase with a significant degree of concern of "falsified images" were identified in published articles over a period of 20 years [1989-2008] in a 2 yearly based analysis(Wright, Titus, \& Cornelison, 2008). Another ORI report disclosed a case of research misconduct by a research coordinator from Emory University in a NHLBI and NIH funded grant in 2009(Jamieson). It was observed and simultaneously acknowledged by the research coordinator that patient information was fabricated to increase the number of enrolees in the study while they never existed on ground. Such kinds of scientific misconduct must attract severe penalties. There should be a regulation to identify such frauds and a unified approach should be applied for internationalization of data quality as well as authenticity of research data published.

In Medical Institutes, GLP compliance could be crucial aspect of bringing robustness to data especially when data generated in such Institutes in generally considered soft. Thus, it is necessary for the basic scientists to implement the validation protocols for quality thesis outcome. It will reinforce the quality of research data acceptable to the international standards

\section{FUNDING}

This research did not receive any specific grant from funding agencies in the public, commercial, or not-for-profit sectors.

\section{Acknowledgments}

We would like to express our gratitude towards Dr.Sudhir Srivastava who has helped in the setting up the GLP system in the lab since 2009.

\section{REFERENCES}

1. Best, J. W., \& Kahn, J. V. (2016). Research in education: Pearson Education India.

2. Carson, P. A., \& Dent, N. J. (2007). Good clinical, laboratory and manufacturing practices: techniques for the QA professional: Royal Society of Chemistry.

3. Corti, L., Van den Eynden, V., Bishop, L., \& Woollard, M. (2014). Managing and sharing research data: a guide to good practice: Sage.

4. Crouse, D. D., Coverston, H. G., \& Cychosz, J. M. (1998). Archiving file system for data servers in a distributed network environment. In: Google Patents.

5. Cushman, M., Cornell, E. S. Howard, P. R., Bovill, E. G., \& Tracy, R. P. (1995) Laboratory methods and quality assurance in the Cardiovascular Health Study. Clinical chemistry, 41(2), 264-270.

6. Evans, J. R., \& Lindsay, W. M. (2013). Managing for quality and performance excellence: Cengage Learning.

7. Furht, B., \& Escalante, A. (2010). Handbook of cloud computing (Vol.3): Springer.

8. Grant, D. S., \& MacDonald, S. E. (1986). Matching to element and compound samples in pigeons: The role of sample coding. Journal of Experimental Psychology: Animal Behavior Processes, 12(2), 160

9. Haider, S. I. (2001). Pharmaceutical master validation plan: the ultimate guide to FDA, GMP, and GLP compliance: CRC Press.

10. Hancock, D. R., \& Algozzine, B. (2016). Doing case study research: A practical guide for beginning researchers: Teachers College Press.

11. Hartmann-Fritsch, F., Marino, D., \& Reichmann, E. (2016). About ATMPs, SOPs and GMP: the hurdles to produce novel skin grafts for clinical use. Transfusion Medicine and Hemotherapy, 43(5), 344-352.

12. Herr, K., \& Anderson, G. L. (2014). The action research dissertation: A guide for students and faculty: Sage publications.

13. Hooijmans, C. R., de Vries, R., Leenaars, M., Curfs, J., \& Ritskes-Hoitinga, M. (2011). Improving planning, design, reporting and scientific quality of animal experiments by using the Gold Standard Publication Checklist, in addition to the ARRIVE guidelines. British journal of pharmacology, 162(6), 1259-1260.

14. Horvath, A. R. (2013). From evidence to best practice in laboratory medicine. The Clinical Biochemist Reviews, 34(2), 47

15. Jamieson, J. Case Summaries. Office of Research Integrity, 8

16. Jena, G., \& Chavan, S. (2017). Implementation of Good Laboratory Practices (GLP) in basic scientific research: Translating the concept beyond regulatory compliance. Regulatory Toxicology and Pharmacology, 89, 20-25.

17. Kerzner, H., \& Kerzner, H. R. (2017). Project management: a systems approach to planning, scheduling, and controlling: John Wiley \& Sons.

18. Kiranmai, G. U. Good Laboratory Practice (GLP)

19. Mayer, T., \& Steneck, N. (2007). Final Report to ESF and ORI First World Conference Mayer, T., \& Steneck, N. (2007). Final Report to ESF and ORI Firs

20. McLachlan, S. (2017). Realism in synthetic data generation: a thesis presented in fulfilment of the requirements for the degree of Master of Philosophy in Science, School of Engineering and Advanced Technology, Massey University, Palmerston North, New Zealand. Massey University,

21. Neuroscience Research Lab, D. o. N., Post Graduate Institute of, \& Medical Education and Research, C. (2016). Redefining Quality Standards in BasicResearch Investigations by Broadening thePurview of GLP.

22. Opara, L. U. (2003). Traceability in agriculture and food supply chain: a review of basic concepts, technological implications, and future prospects. Journal of Food Agriculture and Environment, 1, 101-106.

23. Organization, W. H. (2010). Handbook: good laboratory practice (GLP): quality practices for regulated non-clinical research and development: World Health Organization.

24. Paasche-Orlow, M. K., Taylor, H. A., \& Brancati, F. L. (2003). Readability standards for informed-consent forms as compared with actual readability. New England journal of medicine, 348(8), 721-726

25. Panel, B. B. R. (2012). Guidelines for safe work practices in human and animal medical diagnostic laboratories. Morbidity and Mortality Weekly Report, 61

26. Paszko, C., \& Turner, E. (2001). Laboratory information management systems: CRC press.

27. Refaeilzadeh, P., Tang, L., \& Liu, H. (2016). Cross-validation. Encyclopedia of database systems, $1-7$.

28. Sickles, E (1992). Quality assurance. How to audit your own mammography practice. Radiologic Clinics of North America, 30(1), 265-275

29. Taylor, J. K., \& Tranter, R. (1987). Quality assurance of chemical measurements (Vol. 129): Lewis Publishers Chelsea, MI

30. Wassie, M. A., Zeleke, A. A., Dachew, B. A., \& Kebede, M. (2017). Evidence-based practice and its associated factors among medical laboratory professionals in West Amhara hospitals, Northwest Ethiopia. International journal of evidence-based healthcare.

31. Wright, D. E., Titus, S. L., \& Cornelison, J. B. (2008). Mentoring and research misconduct: An analysis of research mentoring in closed ORI cases. Science and Engineering Ethics, 14(3), 323-336. 